\title{
Noise and Blood Pressure: a Cross Sectional and Longitudinal Study of the Effects of Exposure to Loud Noise on Residents in Calabar, Cross River State, Nigeria
}

\author{
Ubon E. Asuquo, Michael U. Onuu and Aniefiok O. Akpan \\ Department of Physics, University of Calabar, P.M.B. 1115, Calabar, Cross River State, Nigeria.
}

\author{
Affiong U. Asuquo \\ Department of Anaesthesiology, University of Calabar Teaching Hospital, Calabar, Cross River State, Nigeria.
}

(Received 12 December 2007; accepted 11 March 2009)

\begin{abstract}
A cross-sectional and longitudinal study of the effects of loud noise on residents of Calabar, Nigeria, has been carried out using subjective and objective measures. For the cross-sectional study, a 39-item questionnaire was used for the subjective measure. A sample size of 1000 people in the high noise zones with equivalent continuous A-weighted sound pressure levels over $80 \mathrm{~dB}$ was randomly selected and used as the study group. In the low noise zone with equivalent continuous A-weighted sound pressure levels under $80 \mathrm{~dB}, 1000$ people were also randomly selected and used as the control group. For the longitudinal study, a sample size of 15 persons who had worked in the Calabar Timber Market from July 2002 (when the first blood pressure assessment was carried out) to July 2006 (when the second blood pressure assessment was carried out) was used. Findings show that exposure to loud noise may not have an immediate effect on blood pressure, but, when individuals are exposed for a long period of time (4 years in this study), it leads to a significant rise in blood pressure.
\end{abstract}

\section{INTRODUCTION}

Effects on the systemic circulation, such as the constriction of blood vessels, have been observed under laboratory and field conditions. Many studies have shown blood pressure to be higher in noise-exposed workers and in populations living in noisy areas around airports and on noisy streets than in control populations. Other investigations indicate no blood pressure effects. The overall evidence suggests that a weak correlation exists between long-term noise exposure and blood pressure elevation, or hypertension. More research is required in order to estimate the long-term cardiovascular and psychophysiological risks due to noise. In real life, community noise interferes with a number of activities, for example recreation, sleep, communication, and concentration. The risk of adverse effects on health must be considered in the light that noise as a stressor may operate through physiological responses modified in complex ways by individual psychological processes.

In field and laboratory experiments, Ising $^{1}$ finds no correlation between human blood pressure variation and noise exposure (traffic noise played back at $60 \mathrm{~dB} \mathrm{~L} \mathrm{Amax}_{\text {max }}, 6 \mathrm{~h}$, and intermittent white noise at $100 \mathrm{~dB} \mathrm{~L}_{\mathrm{Amax}}, 5 \mathrm{~min}$, respectively). It seems that direct effects of short-term exposure to loud levels of noise have little to do with long-term exposure which interferes more with daily activities. Nevertheless, short-term noise-induced disturbances may be associated with the same type of stress reactions which are described as part of the general adaptation syndrome. ${ }^{2,3}$

Studies suggest that noise-induced stress may increase the excretion of magnesium which may cause a magnesium imbalance, especially when dietary magnesium intake is marginal. ${ }^{4}$ Serum magnesium deficiency, in turn, may produce progressive vasoconstriction, vasospasm and ischemia which, given time, may lead to hypertension and coronary heart disease. This theory is supported by the fact that a long-term increase of blood pressure has been shown to be negatively correlated to the concentration of intracellular magnesium. Emerging data further suggests that low serum magnesium levels may exacerbate the effects on the blood pressure of prolonged noise exposure. $^{5}$

There may be a genetic basis for cardiovascular reactions to noise in that persons with normal blood pressure, who belong to families with at least one hypertonic family member, seem to react with higher blood pressure elevation in stressful situations than others. ${ }^{6,7}$ Physiological reactivity characteristics have not been sufficiently explored to date.

Noise sensitivity has also been put forward as one of the predictors of cardiovascular response to noise. Subjects who describe themselves as sensitive to noise have reacted to noise with larger increases in vasoconstriction than their "normal" counterparts. Aro ${ }^{8}$ reports that pressure level of noise is a significant predictor of blood pressure change only for subgroups of workers. According to Rehm, ${ }^{9}$ individual responses to noise may be more highly correlated with symptoms of illhealth than with the noise itself. Furthermore, a person's reports about symptoms of ill-health seem to be related to the quality of sleep. Sensitivity to noise is related to reported sleep 